\title{
Variabilité des caractéristiques morphologiques des os du gigot de l'agneau mâle. Relation avec la conformation
}

\author{
M.R. ANOUS * \\ INRA, Laboratoire de Recherches sur la Viande, \\ Centre de Recherches de Jouy-en-Josas, 78350 Jouy-en-Josas
}

\begin{abstract}
Résumé
La variabilité de la forme des différentes pièces osseuses dans le gigot de l'espèce ovine et sa relation avec la morphologie générale du gigot appréciée par la valeur de sa longueur $(F)$ a été étudiée par deux types d'analyses multidimensionnelles (composantes principales et données centrées) et par régression multiple, en considérant un échantillon de 52 agneaux mâles $(19,49 \pm 3,10 \mathrm{~kg}$ de carcasse) représentatif de la variabilité existant dans la population ovine française et comprenant des produits de différents types morphologiques provenant de diverses races pures ou de croisement et abattus à un stade comparable de développement (même état d'engraissement relatif de la carcasse). On a considéré au total 18 mesures osseuses (6 pour le coxal, 6 pour le fémur, 4 pour le tibia et 2 pour le tarse).
\end{abstract}

La variabilité des différentes mesures osseuses est assez voisine pour le tibia et le fémur et un peu plus forte dans le cas de l'os coxal.

Les relations entre les différentes dimensions sont très variables. Les longueurs des os sont très fortement liées entre elles mais sans liaison significative avec les autres mesures des os. Par rapport aux mesures osseuses, $F$ n'est lié qu'avec les longueurs (positivement) et avec la circonférence du fémur (négativement), ce qui implique une relation négative entre la diminution de $F$ (donc l'amélioration de la conformation) et l'allongement des os du gigot.

En considérant l'ensemble des mesures de la forme des os, les analyses successives ont permis de définir une série de rapports impliquant un nombre limité de mesures. On peut distinguer les différents types raciaux par la valeur des rapports des mesures suivantes : longueurs des os (coxal, fémur et tibia), circonférence du fémur, largeur supéricure du corps du fémur, largeur de l'extrémité inférieure du tibia, largeur de l'os ischium et largeur de l'os ilium.

L'analyse des interrelations entre les variables osseuses et la conformation (la valeur F) montre l'importance du squelette dans l'explication de la variation constatée de la conformation chez l'espèce ovine. La sélection sur ce caractère est envisageable en considérant un nombre limité de mesures (longueur du tibia, circonférence du fémur, largeur de l'ischium) qui expliquent, au total, 81 p. 100 de la variation de F.

Mots clés: Mensurations des os, agneau, conformation.

$\left({ }^{*}\right)$ Adresse actuelle : Department of Animal Production, Faculty of Agriculture, Ain-Shams University, PO Box 68 Hadayek Shoubra, 11241 Cairo, Egypte. 


\section{Introduction}

Le squelette est pour l'organisme un système fonctionnel déjà très développé prénatalement et que l'on peut classer comme un tissu de maturité précoce. Il doit satisfaire à deux besoins mécaniques très importants, d'une part en fournissant un support de construction pour l'organisme, et d'autre part, en transmettant la charge pour que le cartilage articulaire irremplaçable des joints synoviaux puisse fonctionner dans l'étendue physiologique des mouvements (Poss, 1984). Les propriétés mécaniques essentielles de l'os sont l'élasticité et la résistance. L'élasticité est faible. La résistance à la fracture dépend de la nature (statique ou cinétique), de la direction et du point d'application de la force exercée (par exemple pression, torsion, tension...). Par ailleurs, c'est un tissu qui ajuste son architecture selon la charge fonctionnelle qu'il doit supporter (RUBIN, 1984), donc suivant le mode de locomotion et suivant la conformation et le poids.

Puisque le squelette appendiculaire des Mammifères, relativement constant quant à sa construction d'ensemble, est très divers dans le détail des formes, les caractères de ses os peuvent traduire les particularités du mode de vie (surtout habitat et locomotion). Par ailleurs, Dumont et Boulleau (1984) et Anous (1986 a) ont suggéré que les variations existant entre animaux dans la forme et les dimensions des diverses pièces de l'ossature sont, en effet, de nature à modifier les possibilités de développement des muscles qu'elles supportent.

Les caractères morphologiques des os des différentes espèces des Mammifères ont été étudiés en détail par les anatomistes dans une vue très descriptive comme le montrent, par exemple, les ouvrages de GeTrY (1975) et BARONE (1980) mais l'aspect quantitatif de la variabilité morphologique des os n'a pas été considéré. Par ailleurs, depuis les premiers travaux de Hammond (1932) chez les ovins sur le développement du squelette en masse et en volume et les différents facteurs responsables de sa variabilité et ensuite les travaux de Pálsson (1940) chez les ovins, la variabilité de la forme des os a été envisagée, essentiellement dans ses implications zootechniques, en considérant le métacarpe et le métatarse (Orme et al., 1959 ; Orts, King et Butler, 1969 ; Coble et al., 1971 ; WILSON et al., 1977 ; WILSON et al., 1982). On manque d'information sur l'amplitude de la variation rencontrée dans ces os comme dans les autres os entre individus présentant des types morphologiques très différents. Par ailleurs, les interrelations entre la forme des diverses pièces osseuses ont été pendant longtemps peu étudiées, faute de méthodes statistiques acceptables. La mise au point des techniques d'analyse multidimensionnelle a permis de pallier les difficultés d'analyse et a été ainsi largement utilisée dans plusieurs études ostéométriques (KARCHOUd, 1981 ; THORPE et LeAmy, 1983 ; Cracraft, 1976).

Le présent travail rapporte les résultats obtenus dans une étude entreprise chez les ovins en considérant les caractéristiques morphologiques des supports osseux du gigot dans un ensemble d'agneaux mâles représentatifs de la variation existant dans la conformation et considérés à des états de développement corporel comparable. La conformation était appréciée ici par la valeur de la longueur du gigot (F) mesurable selon BocCARD et al. (1964). 


\section{Matériel et méthodes}

\section{A. Animaux}

On a considéré au total 52 agneaux mâles choisis comme suit :

- Vingt d'entre eux ont été choisis vivants dans un grand abattoir spécialisé en ovins de manière à constituer un échantillon représentatif des cinq classes de conformation définies dans le système officiel de classement des carcasses, à raison de 4 agneaux mâles pour chacune des classes $\mathrm{E}, \mathrm{U}, \mathrm{R}, \mathrm{O}$ et $\mathrm{P}^{(*)}$.

- Le restant des animaux $(\mathrm{N}=32)$ a été choisi essentiellement pour constituer des groupes de 3 agneaux des races suivantes: Charolaise, Ile-de-France, Rava, Romanov, Southdown, Suffolk, Tarasconnaise, Texel. En plus, on a retenu trois mâles de race Charmoise, deux de race Vendéenne et un de race Rouge-de-l'Ouest, choisis parmi les agneaux participant au concours d'ovins abattus du concours général agricole 1984 ainsi que deux mâles de race Solognote.

Le protocole d'abattage adopté et la méthode de dissection utilisée sont ceux décrits par ANous (1986 a). Sur la carcasse refroidie, on a procédé à la mesure de la longueur $F$ (longueur du gigot en $\mathrm{cm}$ ) comme décrit par Boccard et al. (1964). Le poids moyen de carcasse froide a été de $19,49 \pm 3,10 \mathrm{~kg}$.

Après leur séparation individuelle du gigot, les différentes pièces osseuses ont été mises dans une solution d'hydroxyde de sodium (40 p. 100) pendant 12 heures pour assurer le nettoyage des os qui étaient ensuite rincés à l'eau chaude.

\section{B. Mensurations}

Les mensurations osseuses choisies dans cette étude sont celles qui ont été déjà utilisées par différents auteurs: HAMMOND (1932) ; PÁlsSon (1939) ; CuthBertson et Pomeroy (1962); Latimer et Sawin (1963 a et b) ; Cracraft (1976) ; McHenry (1978) ; Steudel (1981) ; McLain et Vig (1983) ; Lozanoff, Sciulli et Schneider (1985) ; KNudson et al. (1985) ; Anderson, Hall-Martin et Russel (1985)

La figure 1 présente les mensurations étudiées dont l'annexe donne la definition détaillée.

Les mensurations des os étaient effectuées à $0,1 \mathrm{~mm}$ près en utilisant un pied à coulisse pour les dimensions de longueur et de largeur. Les dimensions de circonférence du fémur et du tibia ont été relevées à l'aide d'une ficelle plastique de 1,7 mm de diamètre dont la longueur était ensuite mesurée, après déroulement, à l'aide d'une règle graduée en $\mathrm{mm}$.

\section{Traitement statistique}

La moyenne, l'écart type et le coefficient de variation de chacune des variables ainsi que les corrélations entre les variables ont été calculés.

(*) Cf. Catalogue de classement des carcasses d'ovins de boucherie paru au journal officiel de la République Française du 24 novernbre 1976 p. NC6135. 
Les interrelations existant entre variables, soit de chacun des os coxal, fémur et tibia, soit de l'ensemble des os, ont été analysées par la méthode d'analyse multidimensionnelle des données centrées (LeFEBVRe, 1976). L'ensemble des variables ont été analysées aussi par la méthode d'analyse en composantes principales en travaillant sur la matrice des corrélations. Ensuite les valeurs des variables les plus discriminantes ont été utilisées dans une analyse de régression multiple (SNEDECOR et Cochran, 1967) pour expliquer la variation de la conformation exprimée par la valeur de la longueur du gigot $(\mathrm{F})$.
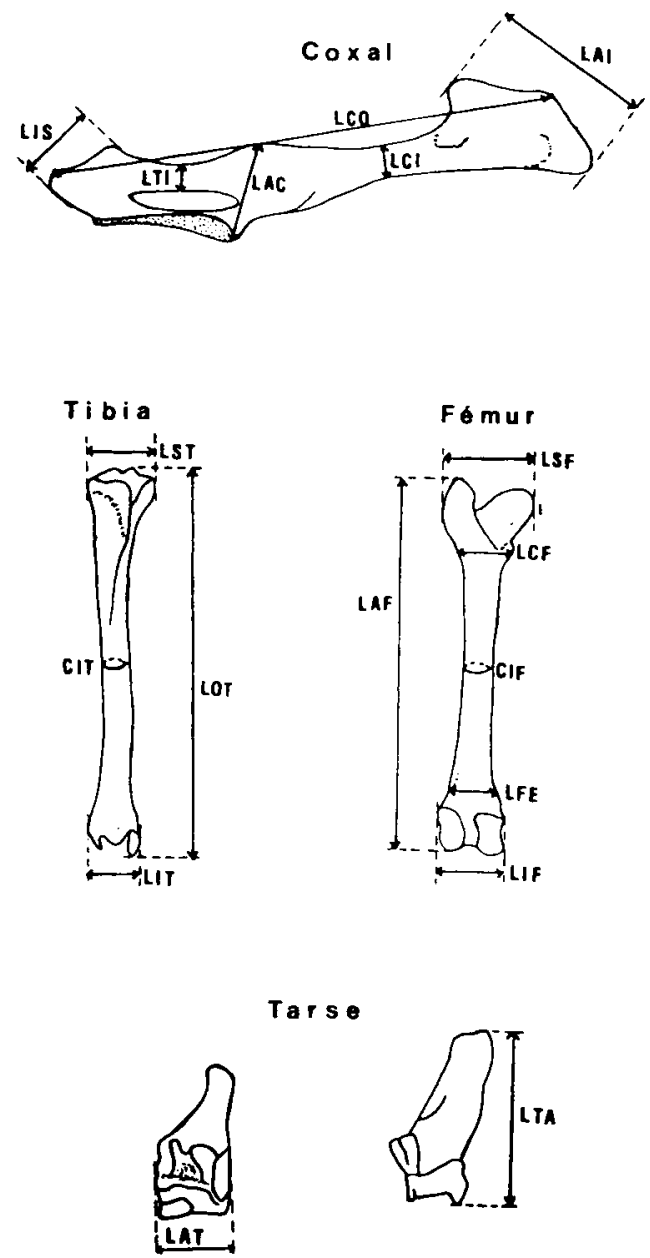

Fig. 1

Mensurations osseuses considérées dans cette étude.

Bone measurements considered in this study. 


\section{Résultats}

\section{A. Variabilité des mesures des os}

Le tableau 1 indique la valeur moyenne, l'écart type et le coefficient de variation pour chacune des mesures ainsi que pour la longueur du gigot $(F)$.

\section{TABleau 1}

Moyenne et écart-type (en cm) et coefficient de variation (en \%) des mesures des os et de la longueur $F(e n \mathrm{~cm})$ du gigot.

Mean and standard deviation (in $\mathrm{cm}$ ) and coefficient of variation (in \%) of bone measurements and $F$ length (in $\mathrm{cm}$ ) of the gigot.

\begin{tabular}{|c|c|c|c|}
\hline $\begin{array}{c}\text { Mesures } \\
\text { Measurements }\end{array}$ & $\begin{array}{l}\text { Moyennes } \\
\text { Mean }\end{array}$ & $\begin{array}{c}\text { Ecart-type } \\
\text { Standard deviation }\end{array}$ & $\begin{array}{c}\text { C.V. } \% \\
\text { Variation coefficient }\end{array}$ \\
\hline $\begin{array}{l}\text { LCO } \ldots \ldots \ldots \\
\text { LAC } \ldots \ldots \ldots \ldots \\
\text { LAI } \ldots \ldots \ldots \ldots \\
\text { LIS } \ldots \ldots \ldots \ldots \\
\text { LTI } \ldots \ldots \ldots \ldots \ldots \\
\text { LCI } \ldots \ldots \ldots \ldots \ldots \\
\end{array}$ & $\begin{array}{r}17,38 \\
5,38 \\
7,03 \\
5,14 \\
1,71 \\
1,75\end{array}$ & $\begin{array}{l}1,22 \\
0,37 \\
0,75 \\
0,51 \\
0,13 \\
0,13\end{array}$ & $\begin{array}{r}7,02 \\
6,86 \\
10,63 \\
9,83 \\
7,59 \\
7,27\end{array}$ \\
\hline 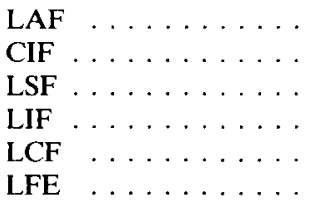 & $\begin{array}{r}16,36 \\
6,21 \\
5,04 \\
4,28 \\
2,81 \\
2,35\end{array}$ & $\begin{array}{l}1,22 \\
0,43 \\
0,34 \\
0,27 \\
0,23 \\
0,20\end{array}$ & $\begin{array}{l}7,46 \\
7,01 \\
6,68 \\
6,33 \\
8,09 \\
8,59\end{array}$ \\
\hline $\begin{array}{l}\text { L.OT } \ldots \ldots \ldots \ldots \\
\text { CIT } \ldots \ldots \ldots \ldots \ldots \\
\text { LST } \ldots \ldots \ldots \ldots \ldots \\
\text { LIT } \ldots \ldots \ldots \ldots \ldots\end{array}$ & $\begin{array}{r}18,84 \\
5,32 \\
4,72 \\
3,31\end{array}$ & $\begin{array}{l}1,62 \\
0,34 \\
0,29 \\
0,25\end{array}$ & $\begin{array}{l}8,59 \\
6,45 \\
6,23 \\
7,70\end{array}$ \\
\hline $\begin{array}{l}\text { LTA } \ldots \ldots \ldots \\
\text { LAT } \ldots \ldots \ldots \ldots\end{array}$ & $\begin{array}{l}6,63 \\
2,81\end{array}$ & $\begin{array}{l}0,47 \\
0,21\end{array}$ & $\begin{array}{l}7,05 \\
7,38\end{array}$ \\
\hline $\mathbf{F} \ldots \ldots \ldots \ldots$ & 26,08 & 3,38 & 12,96 \\
\hline
\end{tabular}

Les valeurs des coefficients de variation montrent une variabilité du même ordre de grandeur pour les différentes mesures des os et vont de 6,23 à 10,63 p. 100 pour les dimensions LST et LAI, respectivement. La variabilité moyenne est de 8,20 p. 100, 7,36 p. $100,7,24$ p. 100 et 7,21 p. 100 pour l'os coxal, le fémur, le tibia et le tarse, respectivement.

D'une façon générale, la variabilité des mesures des os est plus faible que celle de la longueur de gigot $(\mathrm{F})$. 


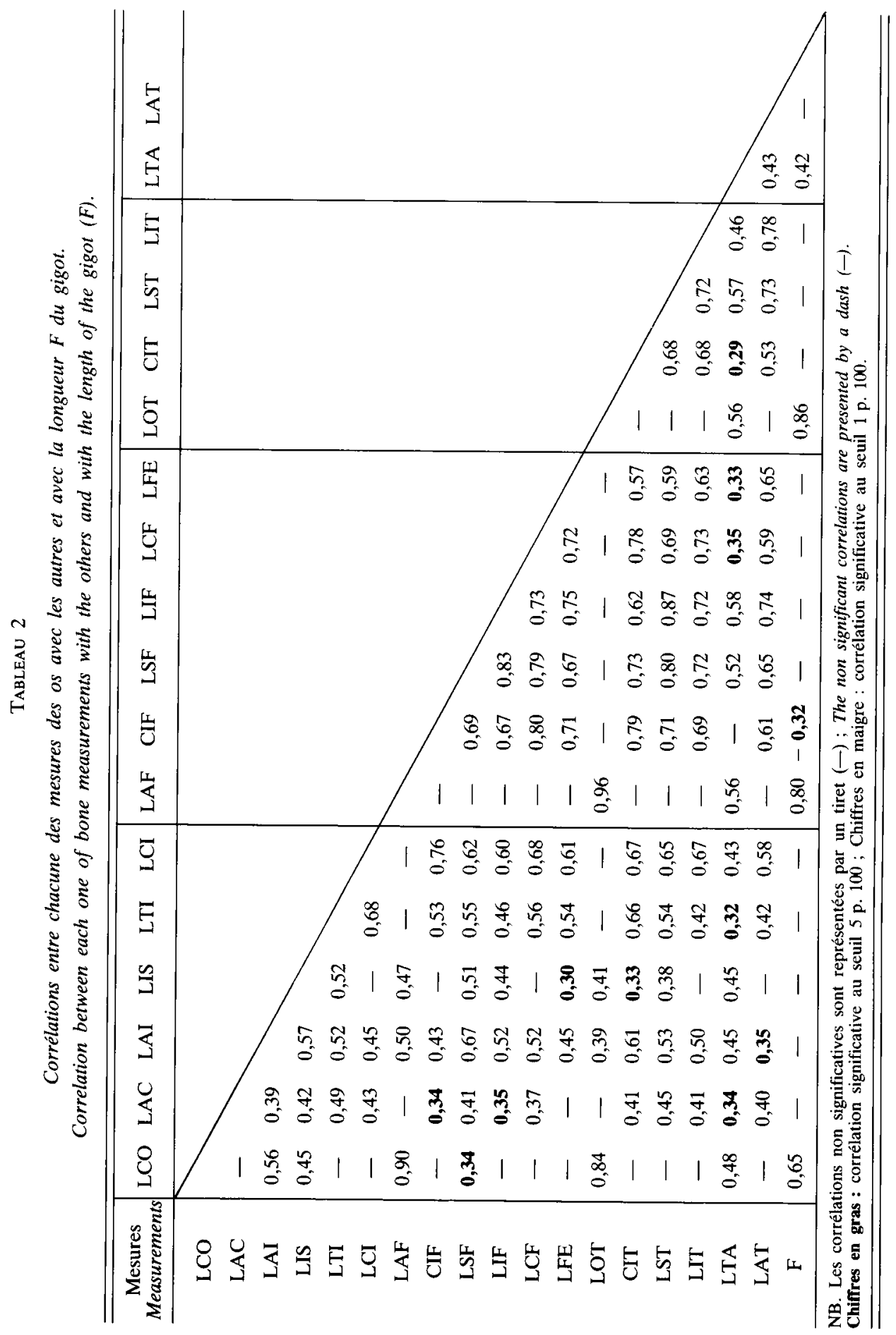




\section{B. Relations entre les différentes mesures des os}

\section{Corrélations simples}

Il existe une grande variabilité dans la valeur des coefficients de corrélation entre les dimensions des différents os du gigot. Sur l'ensemble des 153 relations on observe qu'un certain nombre ( $N=42$, soit 27,4 pour cent des corrélations) ne sont pas significatives au seuil 5 p. 100 et ne seront donc pas représentées au tableau 2.

Elles concernent essentiellement les relations faisant intervenir les mesures LCO, LAF, et LOT avec les autres. Les corrélations qui ne sont significatives qu'au seuil 5 p. 100 représentent 7,18 p. 100 de l'ensemble des relations et concernent les relations de LCO avec LSF, LAC avec CIF, LIF et LTA, LAI avec LAT, LIS avec LFE et CIT et LTA avec LTI, LCF, LFE et CIT.

Les autres corrélations $(65,42$ p. 100$)$ sont hautement significatives au seuil 1 p. 100. Parmi elles un faible nombre de corrélations sont finalement d'un niveau très élevé ; 5 seulement sont supérieures à $+0,82$.

En considérant la valeur moyenne des corrélations des dimensions intra os on constate que les moyennes des corrélations sont, également, très différentes et que c'est au niveau de l'os fémur que les moyennes sont les plus élevées $(+0,74)$. Pour les autres os les corrélations entre dimensions sont encore assez élevées pour le tibia $(+0,69)$ mais plus faibles pour l'os coxal $(+0,50)$ et pour le tarse $(+0,43)$. Ceci montre qu'une part de la variabilité de la forme de l'os est spécifique de sa nature propre.

On note, également, que LAI et LIS sont les seules mesures de l'os coxal qui montrent une liaison avec sa longueur LCO. Par ailleurs aucune liaison n'a été trouvée entre les mesures du fémur et sa longueur LAF ainsi qu'entre les mesures du tibia et sa longueur LOT. Par contre, les longueurs des os montrent une relation positive très étroite entre elles, et moins forte avec la longueur du tarse LTA.

Il ressort des résultats du tableau 2 que seules les longueurs des différents os montrent une relation positive hautement significative $(P<0.01)$ avec la longueur du gigot $(F)$. Cependant aucune liaison n'a été trouvée entre $F$ et toutes les autres mesures de largeur et d'épaisseur des os, à l'exception de la circonférence CIF du fémur (négatif et significatif au seuil 5 p. 100). L'allongement du gigot s'accompagne donc d'une augmentation de $\mathrm{F}$ et d'une diminution de CIF.

\section{Interrelations entre les mesures des os}

Le tableau 3 indique le pourcentage de la variation expliquée par les axes dans les différentes analyses des données centrées ainsi que dans l'analyse en composantes principales et précise la nature des variables les plus discriminantes.

a) Os coxal

La figure 2 montre la projection des mesures de l'os coxal sur les deux premiers axes qui expliquent respectivement 37,42 et 23,59 p. 100 de la variation. On trouve une opposition sur l'axe 1 entre deux groupes des variables, (LCO, LAI, LIS) d'un côté et (LCI, LAC, LTI) de l'autre. 


\section{TABLEAU 3}

Part de la variation expliquée par les axes et variables les plus discriminantes dans les différentes analyses.

Part of the variation explained by axes and the most discriminant variables in the different analyses.

\section{\begin{tabular}{l} 
Axes \\
\hline I - Explication de la variation \\
- Analyse des données centrées \\
Centred data analysis
\end{tabular}}

a) Os coxal

- Pourcentage d'explication de chacun des axes ................... Percentage of explanation of each one of the axes

- Pourcentage cumulé Cumulated percentage

b) Fémur

- Pourcentage d'explication de chacun des axes .........................

- Pourcentage cumulé ..........

c) Tibia

- Pourcentage d'explication de chacun des axes . . . . . . . . . . .

- Pourcentage cumulé

d) Ensemble des mesures All measurements

- Pourcentage d'explication . . . . . . . .

- Pourcentage cumulé

e) Ensemble des mesures avec la valeur $\mathrm{F}$ All measurements with $F$ value

- Pourcentage d'explication . . . . . . . .

- Pourcentage cumulé ..........

- Analyse en composantes principales Principal components analysis

Ensemble des mesures

All measurements

- Pourcentage d'explication .........

- Pourcentage cumulé

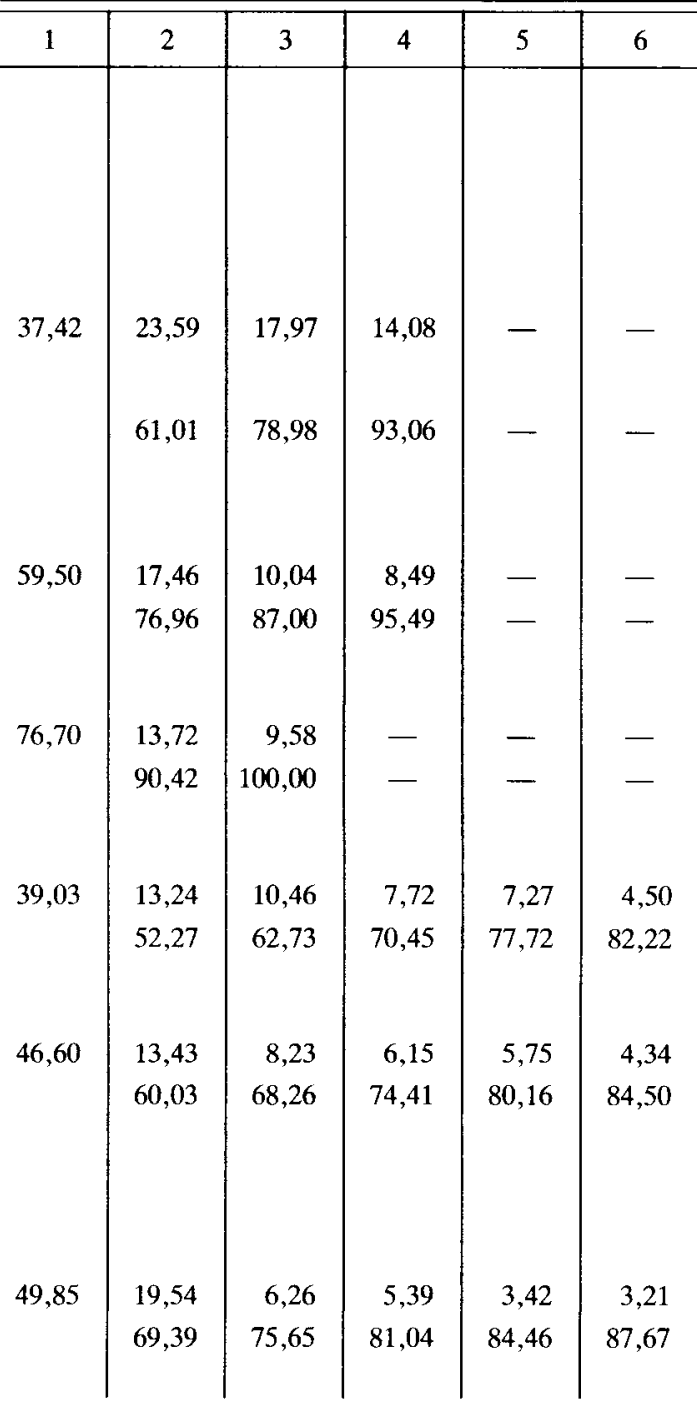


Tableau 3 (suite)

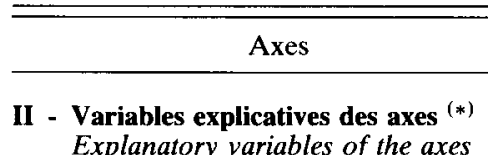

Explanatory variables of the axes

- Analyse des données centrées Centred data analysis

a) Os coxal

$\ldots \ldots \ldots \ldots$

b) Fémur<smiles>CCCCCCCCCCCCCCCCCCCC</smiles>

c) Tibia

$\ldots \ldots \ldots$

d) Ensemble des mes
All measurements

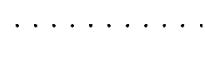

e) Ensemble des mesures avec la valeur $F$ All measurements with $F$ value

- Analyse en composantes principales Principal components analysis

Ensemble des mesures All measurements

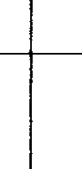

1

T2

$+$

$\mid+4$

\begin{tabular}{|l|l|}
\hline 5 & 6 \\
\hline
\end{tabular}




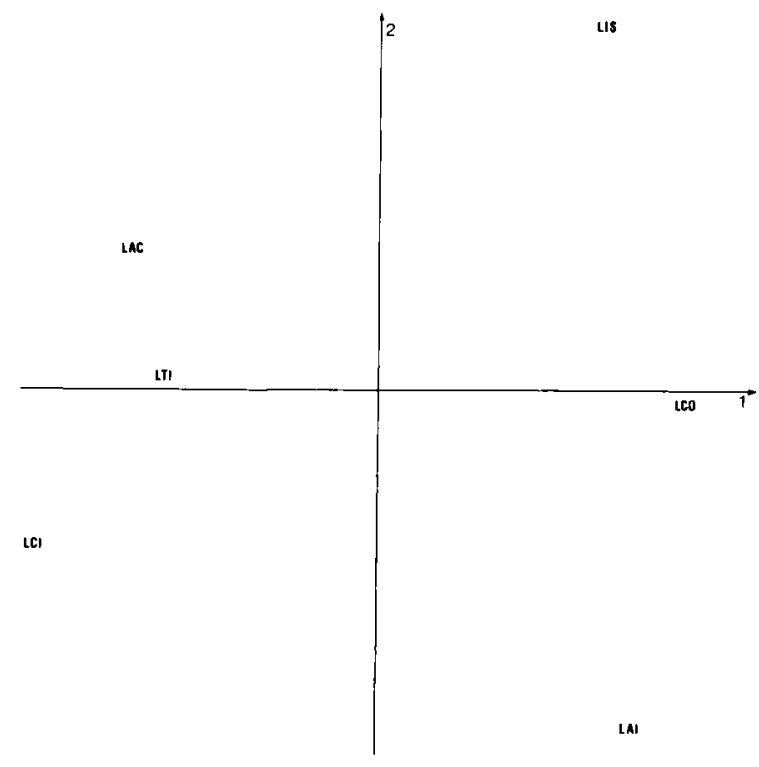

Fig. 2

Plan de projection des mesures de l'os coxal sur les deux premiers axes dans l'analyse des données centrées.

Plane of projection of coxal bone measurements on the first two axes in the centred data analysis.

On observe une opposition principale sur l'axe 2 entre LIS et LAI et une opposition secondaire entre LAC et LCI ; LCO et LTI n'interviennent pas dans cette opposition.

La projection des variables sur les autres axes (non présentée ici graphiquement) montre une opposition sur l'axe 3 entre LCO et l'ensemble (LAI, LIS) et sur l'axe 4 entre LAC et LTI qui expliquent respectivement 17,97 et 14,08 p. 100 de la variation entre animaux.

La part relativement faible des deux premiers axes suggère qu'il n'existe pas de variables majeures, hautement discriminantes par rapport aux autres, et que pour structurer totalement la population, il doit exister une série d'équilibres successifs entre variables.

Dans l'espace des trois premiers axes, qui expliquent plus des trois quarts de la variation de la population, la position des variables les plus discriminantes permet de distinguer nettement des axes de classement des individus sur la base de différents rapports, spécialement de LCO/LCI, LIS/LCI, LAI/LIS, LCO/LAI et LCO/LIS.

Les individus occupent largement l'espace de projection sur les trois premiers axes (non présentés ici faute de place). Pour la plupart et malgré une variabilité intra-groupe quelquefois importante, les groupes raciaux peuvent se distinguer les uns des autres. 
Par exemple les Texel se distinguent des Tarasconnais et des Southdown par des valeurs plus faibles du rapport LCO/LCI (ou LIS/LCI). Les Tarasconnais et les Southdown se distinguent par la valeur du rapport LCO/LAI (ou LCO/LIS) plus élevé chez les premiers.

b) Fémur

La figure 3 montre la projection des mesures du fémur sur les deux premiers axes qui expliquent respectivement 59,50 et 17,46 p. 100 de la variation entre individus.

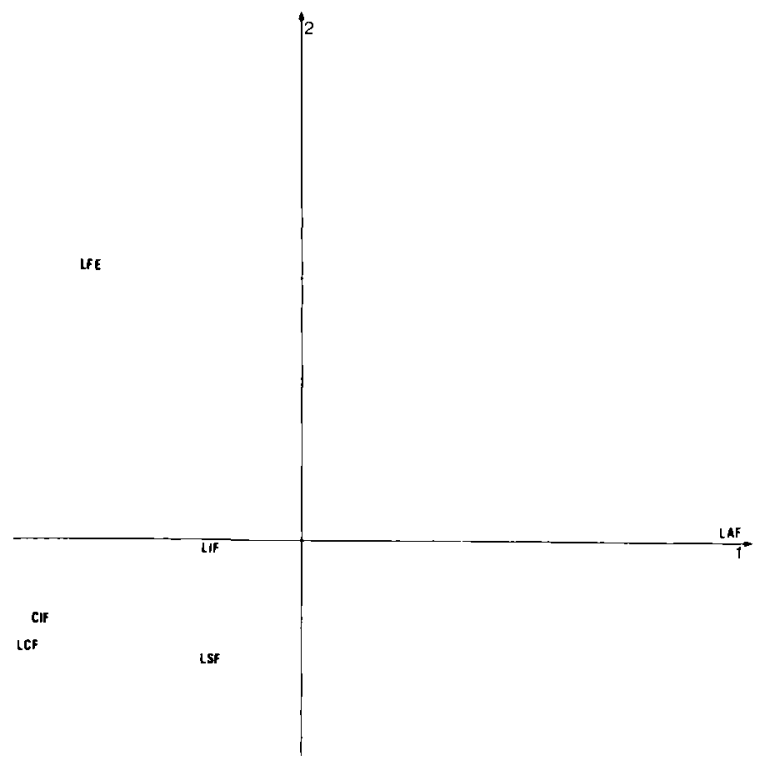

Fig. 3

Plan de projection des mesures du fémur sur les deux premiers ax dans l'analyse des données centrées.

Plane of projection of femur measurements on the first two axes in the centred data analysis.

La part relativement forte des deux premiers axes conduit à considérer les variables les plus discriminantes sur ces axes comme structurant la population d'agneaux (à savoir LAF sur l'axe 1 et LFE sur l'axe 2).

Dans le plan de projection des deux premiers axes on note une opposition sur l'axe 1 entre LAF et toutes les autres dimensions du fémur, spécialement LCF et CIF et sur l'axe 2 entre LFE et l'ensemble (LSF, LCF, CIF). LAF et LIF n'interviennent pas dans cette opposition. On trouve cependant une opposition sur l'axe 3 entre CIF et l'ensemble (LIF, LSF) et sur l'axe 4 entre LCF et l'ensemble (LIF, CIF) qui expliquent respectivement 10,04 et 8,49 p. 100 de la variation de la population (ces deux axes ne 
sont pas présentés ici). Ceci permet de définir des axes particuliers de classement des individus à partir des valeurs des rapports : LAF/LCF, LAF/CIF, LFE/LSF, CIF/LIF et LCF/LIF.

Les individus sont répartis dans l'espace de projection des trois premiers axes (non présentés ici faute de place) et il est possible de séparer les groupes raciaux les uns des autres. Par exemple les Suffolk et les Charmois se distinguent par la valeur du rapport CIF/LIF (ou CIF/LSF), plus élevé chez les deuxièmes.

\section{c) Tibia}

Dans le plan de projection des deux premiers axes (fig. 4) la disposition des variables montre une opposition triangulaire entre LOT, LIT et CIT (LIT et CIT s'opposant à LOT sur l'axe 1 et LIT et CIT s'opposant sur l'axe 2), ce qui définit des axes particuliers de classement des animaux: LOT/LIT, LOT/CIT et LIT/CIT. On observe aussi une opposition secondaire sur l'axe 1 entre LOT et LST. Ceci montre l'importance de la longueur LOT comme variable déterminante de l'axe 1.

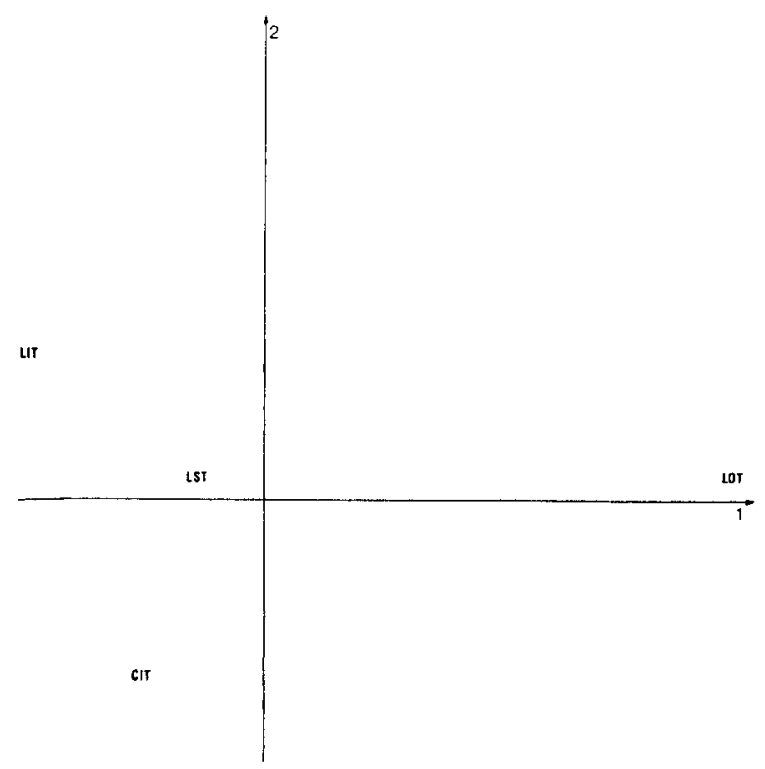

FIG. 4

Plan de projection des mesures du tibia sur les deux premiers axes dans l'analyse des données centrées.

Plane of projection of tibia measurements on the first two axes in the centred data analysis. 
La forte part du premier axe $(76,70 \mathrm{p} .100)$ conduit à considérer les variables les plus discriminantes (à savoir LOT et LIT) comme structurant la population. On trouve cependant une opposition sur l'axe 3 (non présenté ici) entre LST et l'ensemble (LIT, CIT) qui explique 9,58 p. 100 de la variation de la population.

Les individus occupent l'espace de projection sur les trois premiers axes (non représentés ici faute de place) et il est possible de séparer les groupes raciaux les uns des autres sur la base de la valeur des rapports entre les variables déterminant l'axe 1 . Par exemple les Ile-de-France se distinguent des Solognot par des valeurs du rapport LOT/LIT, plus faible chez les premiers.

d) Ensemble des mesures des os

- L'analyse des données centrées

Les figures $5 \mathrm{a}$ et $5 \mathrm{~b}$ indiquent sur les deux premiers axes, qui expliquent respectivement 39,03 et 13,24 p. 100 de la variation entre animaux, la projection de l'ensemble des mesures considérées dans cette étude $(\mathrm{N}=18)$ et des individus.

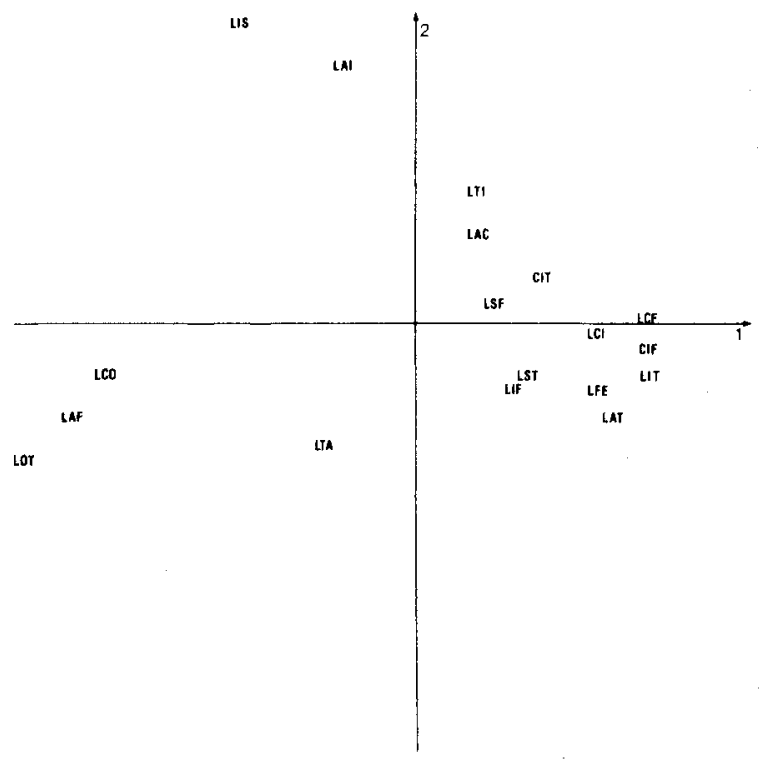

Fig. 5a

Plan de projection de l'ensemble des mesures des os sur les deux premiers axes dans l'analyse des données centrées.

Plane of projection of all bone measurements on the first two axes in the centred data analysis. 


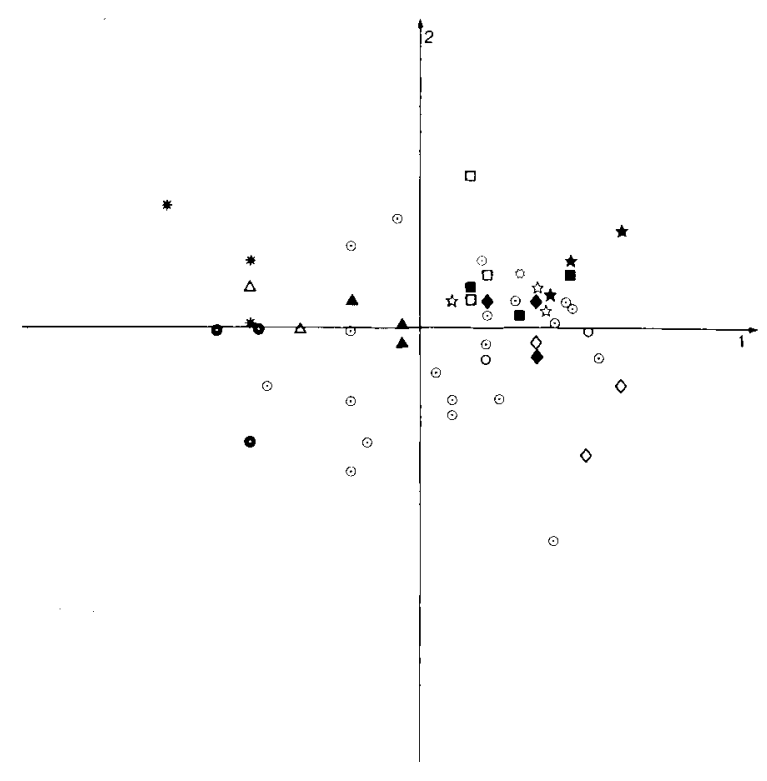

FIG. $5 b$

Plan de projection de l'ensemble des animaux sur les deux premiers axes dans l'analyse

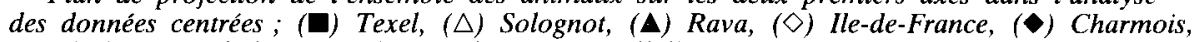

(b) Rouge-de-l'Ouest, (O) Vendéen, ( $\star$ ) Suffolk, (ㄴ) Charolais, (○) Tarasconnais,

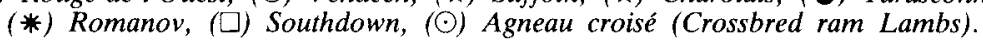

Plane of projection of the animals on the first two axes in the centred data analysis.

Dans le plan de projection des deux premiers axes (fig. 5 a) la disposition des variables montre une opposition triangulaire entre trois groupes des variables : l'ensemble des longueurs (LOT, LAF, LCO) et l'ensemble (CIF, LCF, LIT, LFE, LAT) sur l'axe 1 et ces deux derniers groupes s'opposant à l'ensemble (LIS, LAI) sur l'axe 2. On remarque en plus sur l'axe 2 une opposition entre LTA et l'ensemble (LIS, LAI). Cette situation montre donc l'opposition existant entre, d'une part, les longueurs et, d'autre part, les largeurs et les épaisseurs des os longs et du tarse, c'est-à-dire entre les variables qui traduisent la robustesse des os, d'un côté, et entre ces variables et les variables qui traduisent la largeur du bassin, de l'autre côté.

La projection des variables sur les autres axes (non présentés ici) montre une opposition sur l'axe 3 entre LAI et l'ensemble (LAC, LIS) et sur l'axe 4 entre LTI et l'ensemble (LIT, LAT, LAI, LTA) qui expliquent respectivement 10,46 et 7,72 p. 100 de la variation de la population.

La part relativement forte du premier axe, par rapport aux autres, suggère le rôle important des variables les plus discriminantes comme structurant la population d'agneaux, ce qui définit des axes de classement des individus sur la base des rapports $\mathrm{LOT} / \mathrm{CIF}, \mathrm{LOT} / \mathrm{LCF}, \mathrm{LOT} / \mathrm{LIT}, \mathrm{LAF} / \mathrm{CIF}, \mathrm{LAF} / \mathrm{LCF}, \mathrm{LAF} / \mathrm{LIT}, \mathrm{LCO} / \mathrm{CIF}, \mathrm{LCO} /$ LCF et LCO/LIT. 
Les individus occupent largement l'espace de projection sur les deux premiers axes (fig. $5 \mathrm{~b}$ ) et, pour la plupart, les groupes raciaux peuvent se distinguer les uns des autres. Par exemple les Suffolk et les Ile-de-France se distinguent des Romanov par des valeurs plus faibles des rapports LOT/LIT et LAF/CIF. Les Suffolk et les Ile-deFrance se distinguent par la valeur du rapport LIS/CIF, plus élevé chez les premiers.

- L'analyse en composantes principales

Dans l'espace de projection des deux premiers axes (fig. 6 a) la disposition des variables montre l'opposition entre les longueurs (LAF, LOT, LCO) et toutes les autres dimensions qui expliquent respectivement 49,85 et 19,54 p. 100 de la variation de la population. Il ressort aussi de l'analyse que les variables déterminantes de l'axe 1 sont les mesures de largeur et d'épaisseur des os fémur et tibia. Les variables déterminantes de l'axe 2 sont les mesures de longueur LAF, LOT et LCO. Le regroupement des variables sur un côté de l'axe 1 montre l'importance du facteur taille dans l'explication de la variation entre animaux.

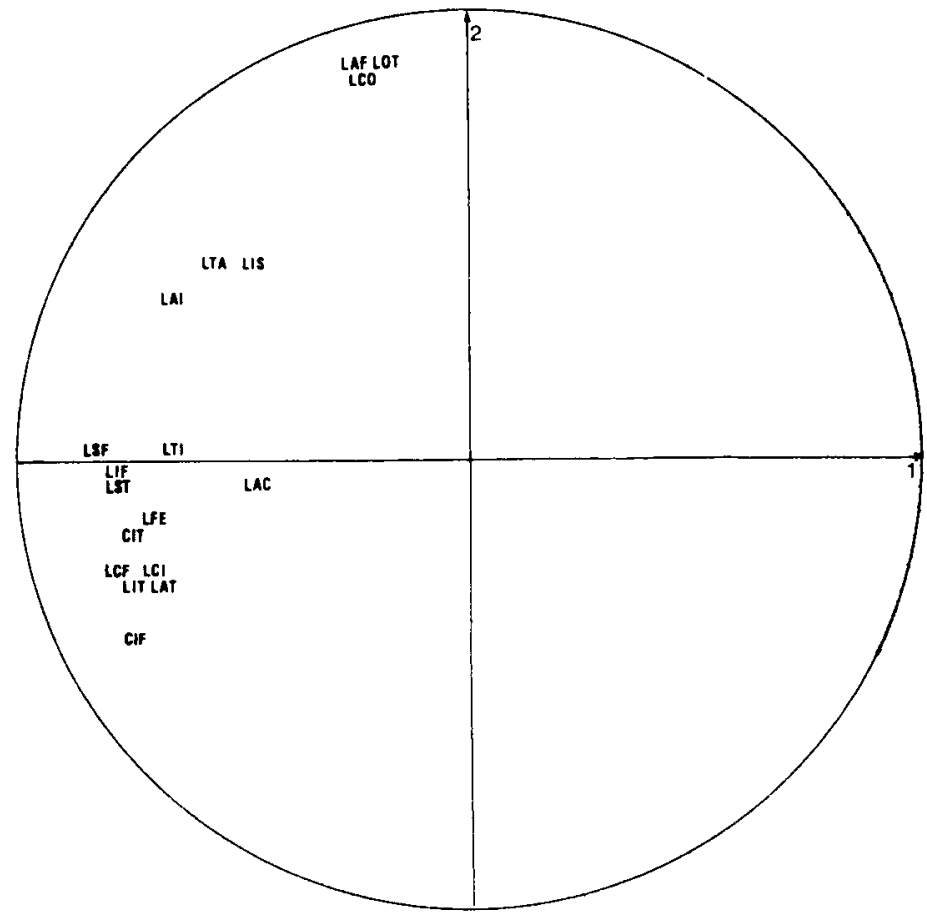

Fig. 6a

Plan de projection, dans un cercle de rayon égal à un, de l'ensemble des mesures des os sur les deux premiers axes dans l'analyse en composantes principales.

Plane of projection, in one circle whose radius equals one, of all bone measurements on the first two axes in the principal components analysis. 
Sur les autres axes (non présentés ici) on note une opposition sur l'axe 3 entre deux groupes des variables : l'ensemble (LAC, LTI, LIS) et l'ensemble (LIF, LAT, LFE, LTA) et sur l'axe 4 entre CIT et l'ensemble (LAC, LTA) qui expliquent respectivement 6,26 et 5,39 p. 100 de la variation. Les variables déterminant ces deux axes sont respectivement LAC, LTI et LIS pour l'axe 3 et LAC et LTA pour l'axe 4. Les rapports entre les variables les plus discriminantes peuvent définir des axes particuliers de classement des individus.

Les individus sont très dispersés dans l'espace de projection des axes 1, 2 (fig. 6 b) et des axes 1, 3 (non présentés ici).

Malgré une variabilité intra-groupe quelquefois importante, les groupes raciaux se distinguent les uns des autres. Par exemple, les Tarasconnais et les Romanov se distinguent des Charmois et des Texel par des valeurs plus fortes du rapport LAF/CIF. On note aussi la position intéressante des animaux Charmois parmi les autres animaux. Cette race se caractérise à la fois par des os courts et minces.

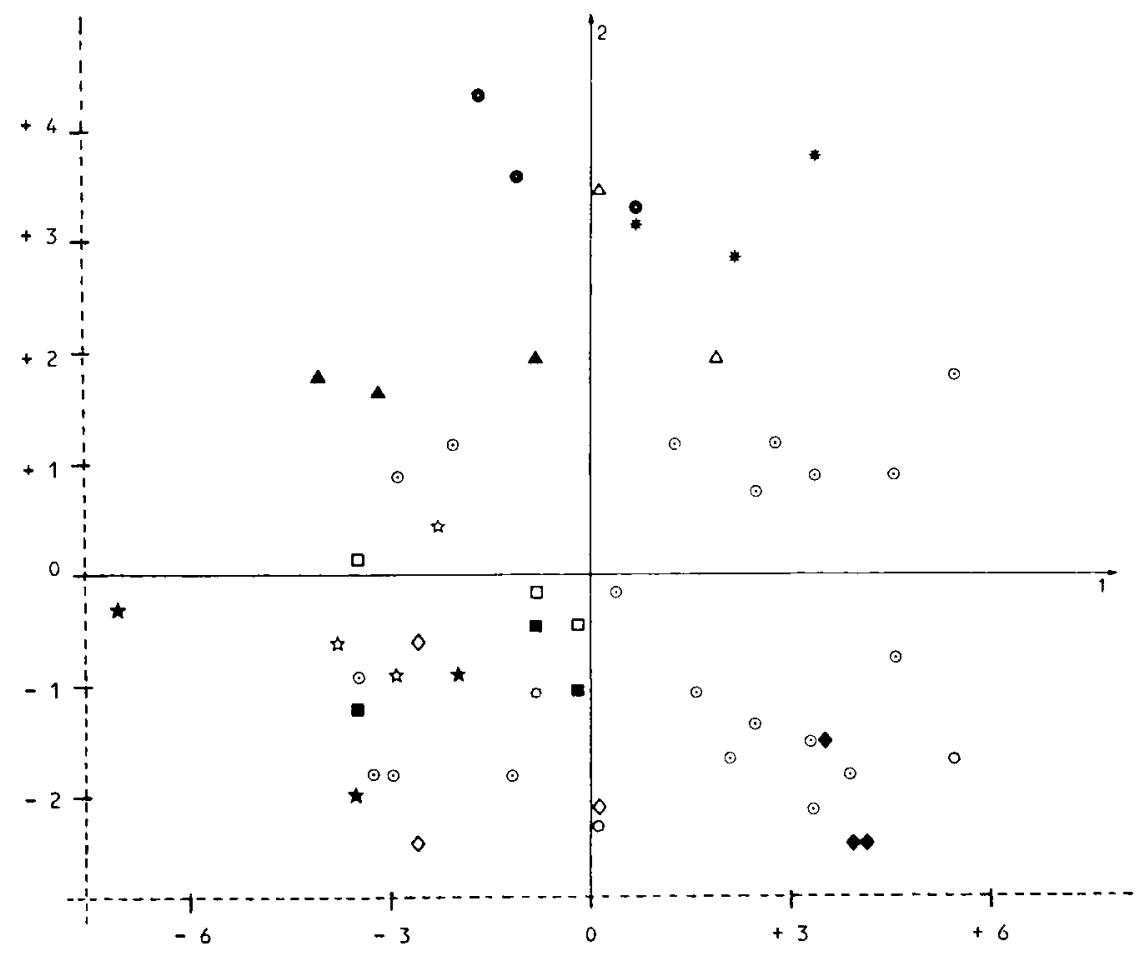

FIG. 6b

Projection de l'ensemble des animaux dans le plan des deux premières composantes principales;

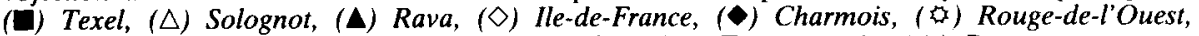
(O) Vendéen, ( $\star$ ) Suffolk, ( $)$ Charolais, (๑) Tarasconnais, (*) Romanov, (口) Southdown, ( $\odot)$ Agneau croisé (Crossbred ram Lambs).

Projection of individuals in the plane defined by the two first principal components. 


\section{e) L'ensemble des mesures des os avec la longueur du gigot}

Pour mieux préciser les interrelations existant entre la morphologie générale du gigot, exprimée par sa longueur $\mathrm{F}$, et la forme des différentes pièces osseuses, la longueur du gigot a été ajoutée à l'ensemble des dimensions et l'analyse des données centrées a été ensuite pratiquée.

On observe une évolution de l'explication de l'inertie de la population sur l'axe 1 et une diminution de la part des axes 3,4 et 5 par rapport à l'analyse précédente. L'essentiel de la variation est donc expliquée par les variables explicatives de l'axe 1 (tabl. 3).

La figure 7 indique la projection de l'ensemble des variables avec l'ensemble des animaux sur les deux premiers axes qui expliquent 46,60 et 13,43 p. 100 de la variation.

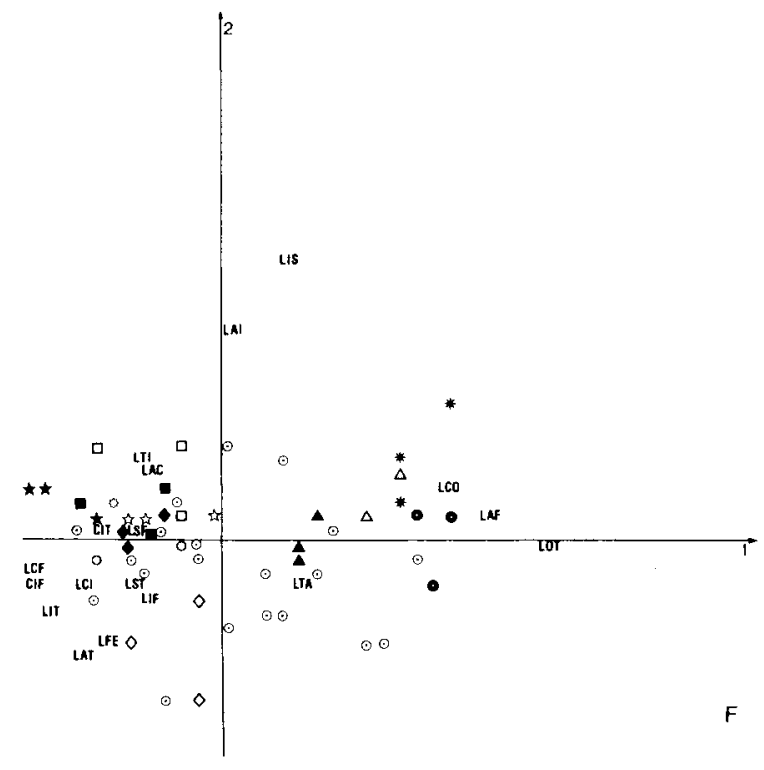

FIG. 7

Plan de projection de l'ensemble des mesures avec l'ensemble des animaux sur les deux premiers axes dans l'analyse des données centrées; (ष) Texel, $(\triangle)$ Solognot, (\) Rava,

$(\diamond)$ Ile-de-France, ( $\bullet$ Charmois, ( $(*)$ Rouge-de-l'Ouest, (O) Vendéen, ( $\star$ ) Suffolk,

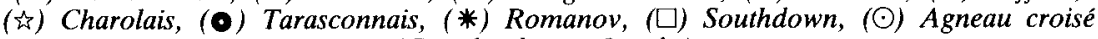
(Crossbred ram Lambs).

Plane of projection of all bone measurements and gigot length $F$ with all animals on the first two axes in the centred data analysis.

L'addition de $F$ a modifié uí peu la position relative d'un nombre limité des variables : LTA, LFE, LIT et CIT sur les axes 1 et 2 et LAI et LIS sur l'axe 3 (non présenté ici) et légèrement la position relative des différents groupes d'animaux, surtout 
celle des Ile-de-France, ce qui conduit à conclure que les variables osseuses peuvent largement expliquer la variation de la conformation.

On observe sur les deux premiers axes une opposition triangulaire entre trois groupes des variables : la longueur du gigot (F) et l'ensemble des mesures de largeur et d'épaisseur du fémur, tibia et tarse et surtout LCF, CIF, LIT, LCI et LAT sur l'axe 1, ces deux groupes s'opposant à l'ensemble (LIS, LAI) sur l'axe 2. La position des longueurs des os autorise à définir également, en plus de ces deux axes principaux, deux autres axes secondaires qui opposent LOT, LAF et LCO aux mêmes groupes des variables que celles qui s'opposent à la longueur $\mathrm{F}$.

La projection des variables sur les autres axes (non présentés ici) montre une opposition sur l'axe 3 entre LAI et LAC et sur l'axe 4 entre LTI et l'ensemble (LTA, LIT, LAT) qui expliquent respectivement 8,23 et 6,15 p. 100 de la variation.

Dans le plan des deux premiers axes, la position des variables les plus discriminantes permet de distinguer nettement la plupart des groupes raciaux sur la base de différents rapports, spécialement de F/LCF, F/CIF, F/LIT, F/LIS et F/LAI.

Pour montrer l'importance des variables les plus discriminantes dans l'explication de la variabilité de la conformation enregistrée entre animaux, on a utilisé dans une analyse de régression multiple différentes variables de mensurations, considérées en valeurs absolues ou dans leur rapport (tabl. 4), pour estimer la valeur de F.

TABleau 4

Valeur des rapports entre les variables.

Ratios between variables.

\begin{tabular}{l|c|c|c|c|c|c}
\hline \hline & \multicolumn{7}{|c}{ Rapports (ratios) } \\
\hline & $\frac{(\text { LAF + LOT) }}{\text { CIF }}$ & $\frac{(\text { LAF + LOT })}{\text { LIT }}$ & $\frac{(\text { LAF + LOT })}{\text { LIS }}$ & $\frac{(\text { LAF + LOT })}{\text { LAI }}$ & $\frac{\text { LIS }}{\text { CIF }}$ & $\frac{\text { LIS }}{\text { LIT }}$ \\
\hline Moyenne / Mean . . & 5,699 & 10,704 & 6,862 & 5,048 & 0,832 & 1,561 \\
CV \% . . . . . . & 11,56 & 11,67 & 9,38 & 10,74 & 11,30 & 11,40 \\
Minimum . . . . . & 4,732 & 8,947 & 5,722 & 4,218 & 0,585 & 1,134 \\
Maximum ...... & 7,092 & 13,365 & 8,526 & 6,509 & 1,220 & 2,232 \\
\hline \hline
\end{tabular}

L'ensemble des 18 mesures explique 85,1 p. 100 de la variation de $F$ et, à elles seules, les trois variables LOT, CIF et LIS expliquent 80,7 p. 100 de cette variation et LOT seul 74 p. 100.

Les rapports de variables fournissent aussi une part notable d'explication de la variation de la conformation, atteignant 67,4 pour cent pour le groupe des 5 rapports les plus significatifs.

A partir des valeurs de LOT, CIF et LIS, la valeur de la longueur du gigot F est donnée par l'équation de régression suivante :

$$
\mathrm{F}=1,897 \text { LOT }-1,138 \text { CIF }-1,415 \text { LIS }+4,643
$$

Le test de la signification de cette régression conduit à une valeur du ratio $F$ de 65,35 , très hautement significative $(\mathrm{P}<0,001)$. 


\section{Discussion}

L'objectif de ce travail est une contribution à l'étude de la variabilité de la forme des différentes pièces osseuses dans le gigot de l'espèce ovine et ses relations avec la morphologie externe du gigot. Cette variabilité a été étudiée entre animaux de différentes races et entre produits de croisement dont les tailles étaient différentes. On a retenu de considérer dans l'échantillon des agneaux mâles d'état relatif de développement corporel voisin, en choisissant des animaux de 3 à 6 mois d'âge dont le poids d'abattage est de l'ordre de 40 p. 100 du poids adulte.

La variabilité enregistrée dans la forme des différents os du gigot est moins importante que de celle de la longueur $F$ (qui traduit le niveau de la conformation). On peut donc s'attendre à ce que $F$ joue un rôle plus important que celui de différentes mesures des os pour discriminer les différents groupes raciaux dans l'analyse multidimensionnelle utilisée dans ce travail. On note cependant que la variabilité des différentes dimensions est comparable et qu'elle est dans l'ensemble un peu plus forte dans le cas de l'os coxal, ce qui pourrait s'expliquer par la structure complexe de cet os ; le coxal est un os plat, de forme très irrégulière et composé de trois constituants : l'ilium, le pubis et l'ischium. Cette situation a été déjà trouvée par LATIMER et SAwIN (1963 a et b) chez le lapin et nous amène à conclure que la variabilité constatée doit avoir une origine intrinsèque caractéristique de chacun des os et de son rôle dans le système locomoteur.

On note, d'autre part, des relations très variables entre les dimensions des os. Pour certains les corrélations sont très fortes, comme, par exemple, les liaisons entre les longueurs des os, et d'autres sont non significatives, comme, par exemple, les liaisons entre les longueurs des os longs (fémur et tibia) et toutes les autres mesures des os. Cette indépendance des longueurs qui résume la structure de l'appareil locomoteur conduit à déterminer, au sein de l'ossature du gigot, des rapports entre mesures qui apparaissent être, comme le montre l'analyse des données centrées, des caractères très importants pour expliquer la variation constatée entre individus dans la morphologie de leur os et qui permettent de classer les animaux.

D'une manière générale il n'y a pas de liaison entre $F$ et les caractères de la forme des os, sauf en ce qui concerne les longueurs des os (positivement liées à F) et la circonférence du fémur (négativement liée). Ceci implique une relation positive entre la diminution de $\mathrm{F}$ (donc l'amélioration de la conformation) et la diminution de longueur des os et une relation inverse entre l'allongement du gigot et l'accroissement de la circonférence du fémur. La relation est toutefois limitée entre $\mathrm{F}$ et la longueur du tarse et la circonférence du fémur puisque la variation de LTA et de CIF n'explique finalement que 18 et 10 p. 100 de la variation de la conformation.

En considérant les mesures de la forme de chacun des os du gigot séparément, les analyses successives ont permis de définir une série de rapports impliquant finalement un nombre limité de mesures : LCO, LIS et LAI dans le cas de l'os coxal, LAF, LFE et CIF dans le cas du fémur et LOT et LIT dans le cas du tibia. Ces rapports permettent de positionner les animaux dans l'espace multidimensionnel. Cette importance des mesures des os dans la discrimination intra ou inter espèces a été déjà trouvée par plusieurs auteurs (Cracraft, 1976 ; Jenkins et Camazine, 1977 ; Karchoud 1981, STEudel, 1981). 
Sur l'ensemble des mesures des os, la position relative des variables dans l'espace de projection des trois premiers axes montre l'importance des longueurs et de quelques autres mesures dans la détermination des axes de classement des individus (à savoir CIF, LIT, LCF, LIS, LAI). Il est clair que les animaux sont projetés à l'intérieur d'un espace défini pour chaque axe d'organisation par les minimas et les maximas enregistrés pour les valeurs des différents rapports présentés au tableau 4.

Ces ratios synthétisent les relations existant entre mesures :

1) des os longs considérés dans deux directions, longitudinalement et transversalement [ratios de type A, comme (LAF + LOT)/CIF et (LAF+LOT)/LIT] ;

2) des longueurs des os longs et des largeurs du bassin [ratios de type $\mathrm{B}$, comme $(\mathrm{LAF}+\mathrm{LOT}) / \mathrm{LIS}$ et $(\mathrm{LAF}+\mathrm{LOT}) / \mathrm{LAI}]$

3) des largeurs du bassin et mensurations des os longs considérés transversalement (ratios de type C, comme LIS/CIF et LIS/LIT).

On voit bien ainsi qu'on peut distinguer deux grands groupes d'animaux, un premier qui représente les races à viande (comprenant, dans notre échantillon, les Suffolk, les Texel, les Southdown, les Charolais, les Ile-de-France et les Charmois), qui se distingue d'un deuxième groupe, qui représente les races rustiques (comprenant ici les Tarasconnais, les Romanov et les Solognot), par des valeurs plus faibles des ratios du type $A$.

La séparation de groupes d'animaux selon leur type dans les analyses des données centrées montre que la forme des os est caractéristique de chaque type génétique.

Par exemple, les Suffolk et les Ile-de-France qui se caractérisent par une valeur plus petite des ratios de type $A$, se distinguent entre eux par une valeur plus élevée du ratio de type $\mathrm{C}$ chez les premiers. On peut donc s'attendre à une masse des os plus élevée chez les Suffolk que chez les Ile-de-France. Par contre chez les Romanov et les Tarasconnais, les animaux se caractérisent par une valeur plus grande des rapports de type $A$ et ils se distinguent entre eux par une valeur plus faible du ratio de type $B$ chez les premiers. Les Tarasconnais ont donc des os plus longs et un bassin moins large. Les animaux Rava se trouvent près du centre de projection, et sont donc des animaux dont la forme des os montre une variabilité moyenne. L'analyse en composantes principales a révélé d'autre part les caractéristiques de la race Charmoise qui apparaît comme modèle des animaux à viande par la briéveté et l'étroitesse.

On remarque également que la position des variables de longueur des os est très voisine, ce qui indique que la longueur des os répond à un modèle de variation, par rapport aux autres mensurations, commun aux trois os. Poss (1984) a noté que les extrémités des os longs sont adaptés pour fournir la plus grande surface d'appui possible (donc diminuer la charge par unité de surface) et il est nécessaire que leur forme primaire soit conservée le long de la vie malgré des notables changements de remodelage que subit le corps de l'os avec l'âge ou les changements d'exercice. Ceci explique l'antagonisme trouvé entre les longueurs et les largeurs et les circonférences des os, surtout dans le cas des deux os longs.

L'analyse en composantes principales de l'ensemble des mesures a montré que la variabilité enregistrée dans les dimensions des différents os du gigot s'explique en partie par un effet « taille » des individus. D'autre part, malgré le soin apporté pour comparer des agneaux d'état semblable de développement corporel, il n'est pas possible d'affirmer absolument que les différences constatées entre individus dans la part relative des 
mesures ne peuvent pas, pour une part, être imputées à des différences de maturité des os. Ce point est en cours d'investigation dans notre laboratoire.

L'analyse des interrelations entre les variables osseuses et la conformation (traduite par la valeur F) effectuée par le biais de l'analyse des données centrées et de la régression multiple a mis en évidence l'existence de différents équilibres qui organisent au niveau du gigot - au sein de l'espèce ovine, et pour des agneaux mâles — les relations entre la conformation et la forme des os.

La variation morphologique des os peut donc expliquer l'essentiel de la variation de la morphologie générale du membre. La part non expliquée de la variation de $F$ peut résulter - en dehors des erreurs de mesure associées au relevé de la longueur des gigots ou à la prise des mensurations osseuses - de l'inadéquation partielle des mesures de morphologie osseuse retenues dans cette étude. La forme des os paraît difficile à exprimer avec des mesures simples. Des études ultérieures devraient, à cet égard, préciser l'intérêt des mesures supplémentaires comme les caractéristiques morphologiques des sections (notamment les diamètres), à plusieurs niveaux le long du corps des os longs, ou comme la longueur de l'ischium ou celle de l'ilium.

Quoi qu'il en soit, les résultats de cette étude montrent l'intérêt, pour la sélection sur la morphologie, de la prise en compte d'un nombre limité de variables : longueur du tibia, circonférence du fémur, largeur de l'ischium. Les possibilités d'accéder in vivo à ces mesures offertes par les techniques modernes d'investigation en matière d'anatomie topographique (comme la tomographie ou le scanner) permettent de les retenir comme critères dans les programmes de sélection des ovins pour améliorer leur conformation générale.

Enfin, il serait intéressant d'étudier les conséquences de la variabilité observée sur la physiologie du mouvement des animaux et la biomécanique du membre postérieur, en comparant notamment les sujets des types extrêmes mis en évidence dans ce travail.

\section{Reçu en janvier 1987. Accepté en janvier 1989.}

\section{Remerciements}

Je remercie M. Dumont pour les critiques et les suggestions faites dans la préparation du manuscrit, $M^{\text {He }}$ MARTIN pour la réalisation des illustrations et $M^{\text {me }}$ LE BLANC pour la dactylographie du texte.

Je remercie également pour l'approvisionnement en animaux nécessaires à cette étude M. Hondermark et M. Lecloux (de l'I.T.O.V.I.C.).

\section{Summary}

Variability of the morphological bone characteristics of gigot in lambs. Relationship with conformation

The variability in the shape of the different bones of gigot in lamb and its relationship with the general morphology of gigot, appreciated by the value of its length (F), was studied by two multivariate analyses (principal components analysis and centred data analysis) and by the multiple 
regression analysis using a sample of 52 ram lambs (carcass weight : $19.49 \pm 3.10 \mathrm{~kg}$ ) representative of the existing variability in the French sheep population and including products of different morphological types from different pure breeds or crosses and slaughtered at a comparable stage of development (same relative stage of carcass fatness). A total of 18 bone measurements were considered ( 6 for the coxal bone, 6 for the femur, 4 for the tibia and 2 for the tarsus) (fig. 1 and annexe).

Variability of the different bone measurements considered in this study was uniform, but slightly higher in the case of coxal bone (Table 1).

Relationships between the different bone dimensions were very variable. Bone length measurements showed strong mutual relationships (Table 2 ). $\mathrm{F}$ was significantly correlated only with bone lengths $(r=+0.86$ and +0.80 for tibia and femur length, respectively) and with the femur circumference $(r=-0.32)$, which involves a negative relation between the reduction of gigot length (improvement in conformation) and the lengthening of its bones.

Considering all bone measurements, the successive analyses allowed to define a series of ratios involving a limited number of measurements. The breed groups could be distinguished by the value of ratios of the following measurements : bone lengths (coxal, femur and tibia), femur circumference, superior width of femur shaft, width of the distal extremity of tibia, width of ischium and width of ilium (fig. 5a, 5b, 6a and 6b).

Analysis of the relationships between bone measurements and conformation ( $F$ values) indicated the importance of skeleton dimensions in the explanation of the established variation of gigot morphology in sheep (fig. 7). Thus, selection for this character is possible by considering a limited set of measurements (length of tibia, circumference of femur and width of the ischium) accounting for 81 per cent of the $F$ variation.

Key words: Bone measurements, lamb, conformation.

\section{Références bibliographiques}

Anderson J.F., Hall-Martin A., Russel D.A., 1985. Long-bone circumference and weight in mammals, birds and dinosaurs. J. Zool., Lond., 207, 53-61.

Anous M.R., 1986 a. Variabilité de la répartition du tissu musculaire du membre postérieur de l'agneau. I. Muscles individuels. Reprod. Nutr. Dévelop., 26 (4), 67-91.

ANous M.R., $1986 \mathrm{~b}$. Interrelations entre principaux composants anatomiques, conformation et longueur des os du gigot des ovins. Ann. Zootech., 35 (2), 93-107.

Barone R., 1980. Anatomie comparée des mammifères domestiques. Tome 1 , Ostéologie, $2^{\mathrm{e}}$ édit. Vigot, Paris, $296 \mathrm{pp}$.

Boccard R., Dumont B.L., Peyron C., 1964. Etude de la production de la viande chez les ovins. VIII. - Relations entre les dimensions de la carcasse d'agneau. Ann. Zootech., 13, 367-378.

Coble D.S., Wilson L.L., Simpson M.J., Varela-Alvarez H., Hitchcock J.P., Ziegler J.H., SINK J.D., WATKINS J.L., 1971. Relation of bovine metacarpal and metatarsal characters to growth and carcass characters. Growth, 35, 79-89.

Cracraft J., 1976. The hindlimb elements of the Moas (Aves, Dinornithidae) : a multivariate assessement of size and shape. J. Morph., 150, 495-526.

Cuthbertson A., Pomeroy R.W., 1962. Quantitative anatomical studies of the composition of the pig at 50,68 and $92 \mathrm{~kg}$ carcass weight. II. Gross composition and skeletal composition. $J$. Agric. Sci., 59, 215-223.

Dumont B.L., Boulleau T., 1984. Note sur l'influence du type génétique sur la composition du membre postérieur du porc femelle. Comparaison Landrace Belge, Landrace Français, Piétrain. Journées Rech. porcine en France, 16, 96-102.

GetTY R., 1975, Sisson and Grossman's The anatomy of the domestic animal ; 5th ed., Vol. 1, W.B. Saunders C.O., Philadelphia, London. Toronto, 1257 pp. 
Hammond J., 1932. Growth and development of mutton qualities in the sheep. Oliver and Boyd, Edinburgh. $597 \mathrm{pp}$.

Jenkins F.A., Camazine S.M., 1977. Hip structure and locomotion in ambulatory and cursorial carnivores. J. Zool., Lond., 181, 351-370.

Karchoud A., 1981. Etude de la taxinomie des équidés d'après les mesures squelettales. Les cahiers de l'Analyse des Données, vol. VI, 4, 453-463.

Knudson B.K., Hogberg M.G., Merkel R.A., Allen R.E., Magee W.T., 1985. Developmental comparisons of boars and barrows : II. Body composition and bone development. J. Anim. Sci., 61, 797-801.

Latimer H.B., Sawin P.B., 1963 a. Morphogenetic studies of the rabbit. XXXIV. Weights and linear measurements of the bones of small race $\mathrm{X}$ rabbits compared with large race III. $A \mathrm{~m}$. J. Anat., 113 (2), 235-243.

LATimer H.B., SAwin P.B., 1963 b. Morphogenetic studies of the rabbit. XXXV. Comparison of the weights and linear measurements in normal and heterozygous dwarf rabbits of race $\mathrm{X}$. Anat. Rec., 146 (2), 85-92.

LefeBvre J., 1976. Analyse des Données Centrées. In Introduction aux Analyses statistiques multidimensionnelles. Masson, Paris, 175-183.

Lozanoff S., Sciului P.W., Schneider K.N., 1985. Third trochander incidence and metric trait covariation in the human femur. J. Anat., 143, 149-159.

McHenry H.M., 1978. Fore-and hindlimb proportions in Plio-Pleistocene hominids. Am. J. Phys. Anthrop., 49, 15-22.

McLain J.B., Vig P.S., 1983. Transverse Periosteal sectioning and femur growth in the rat. Anat. Record, 207, 339-348.

Orme L.E., Pearson A.M., Bratzler L.J., Magee W.T., Wheeler A.C., 1959. The muscle-bone relationship in beef. J. Anim. Sci., 18, 1271-1281.

Orts F.A., King G.T., Butler O.D., 1969. Bone muscle relationships in the bovine carcass. $J$. Anim. Sci., 29, 294-297.

PÁlsson H., 1939. Meat qualities in the sheep with special reference to Scottish breeds and crosses. I. J. agric. Sci., 29, 544-626.

PÁlsson H., 1940. Meat qualities in the sheep with special reference to scottish breeds and crosses. II. Part III : Comparative development of selected individuals of different breeds and crosses as lambs and hoggets. J. agric. Sci., Camb., 30, 1-64.

Poss R., 1984. Functional adaptation of the Human locomotor system to normal and abnormal loading patterns. Calcif. Tissue Int., 36, S 155-S 161.

RuBin C., 1984. Skeletal strain and the functional significance of bone architecture. Calcif. Tissue Int., 36, S 11-S 18.

Snedecor G.W., Cochran W.G., 1967. Statistical Methods. 6th ed., Iowa State University Press, Ames. 593 pp.

SteUder K., 1981. Functional aspects of primate pelvic structure : a multivariate approach. Am. J. Phys. Anthrop., 55, 399-410.

ThORPE R.S., LeAmY L., 1983. Morphometric studies in inbred and hybrid house mice (Mus sp.) : Multivariate analysis of size and shape. J. Zool., Lond., 199, 421-432.

Wilson L.L., Roth H.B., Ziegler J.H., Sink J.D., 1977. Bovine metacarpal and metatarsal dimensions : Sex effects, heritability estimates and relation to growth and carcass characteristics. J. Anim. Sci., 44, 932-938.

Wilson L.L., Grieco C.K., Levan P.J., Watkins J.L., 1982. Bovine metacarpal and metatarsal dimensions : comparison of Aberdeen-Angus and Charolais steers slaughtered at three percentages of mature cow weight. Liv. Prod. Sci., 9, 653-663. 


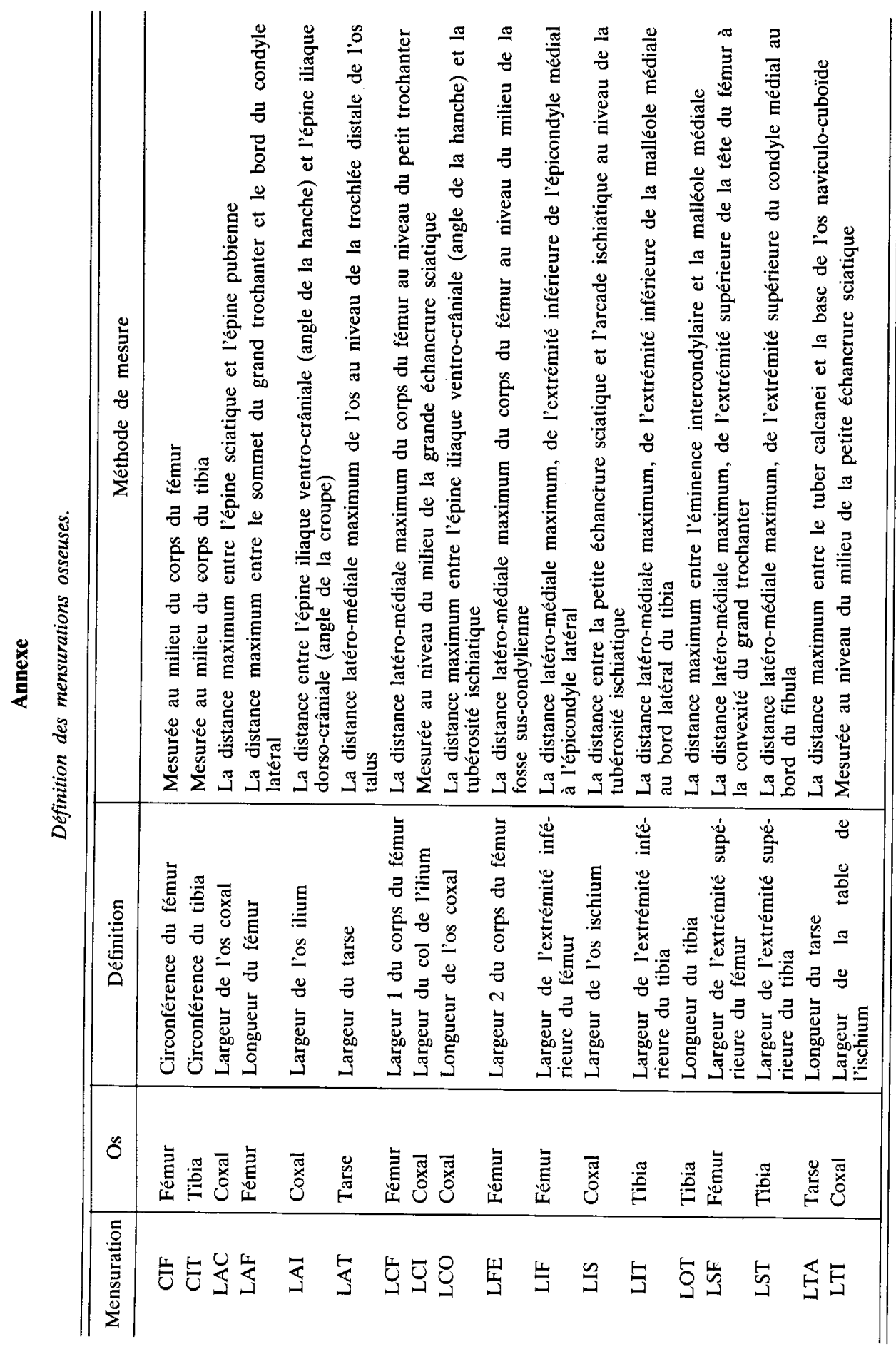

